Nikki Lorraine Y. King-Chao, M.D.' Samantha S. Castaneda, M.D., ${ }^{1,3}$ Michael A. Sarte, M.D. ${ }^{1,2,4}$

\section{'Department of Otorhinolaryngology Head and Neck Surgery \\ The Medical City \\ ${ }^{2}$ Department of Otorhinolaryngology Head and Neck Surgery \\ Rizal Medical Center \\ ${ }^{3}$ Department of Otorhinolaryngology Head and Neck Surgery \\ Jose Reyes Memorial Medical Center}

${ }^{4}$ Department of Otorhinolaryngology Head and Neck Surgery

St. Luke's Medical Center
Correspondence: Dr. Nikki Lorraine Y. King-Chao Department of Otorhinolaryngology - Head and Neck Surgery The Medical City

Ortigas Avenue, Pasig City 1600

Philippines

Phone (632) 6356789 local 6250

Fax (632) 6873349

Email address: nikkilorrainekingmd@yahoo.com

Reprints will not be available from the author.

The authors declared that this represents original material that is not being considered for publication or has not been published or accepted for publication elsewhere, in full or in part, in print or electronic media; that the manuscript has been read and approved by all the authors, that the requirements for authorship have been met by each author, and that each author believes that the manuscript represents honest work.

Disclosures: The authors signed disclosures that there are no financial or other (including personal) relationships, intellectual passion, political or religious beliefs, and institutional affiliations that might lead to a conflict of interest.

Presented at the Clinical Case Report Contest (3rd place), Philippine Society of Otolaryngology - Head and Neck Surgery, Taal Vista Hotel, Tagaytay City, Philippines April 24, 2010.

\title{
Kimura's Disease Initially Diagnosed as Malignancy then Extra-Pulmonary Tuberculosis
}

\begin{abstract}
Objective: To describe a rare case of Kimura's disease initially misdiagnosed as malignancy then tuberculosis.
\end{abstract}

\section{Methods:}

\author{
Design: Case report \\ Setting: Tertiary Private Hospital \\ Patient: One
}

Results: A 30-year-old male with a 6-year history of gradually-enlarging right infra-auricular mass revealed an enlarged mass in the right infraauricular area and multiple cervical lymphadenopathies on physical examination. Initial fine-needle aspiration biopsy was interpreted as pleomorphic adenocarcinoma but succeeding work-ups and imaging studies led to treatment for tuberculosis. Subsequent biopsies finally led to the proper histopathologic diagnosis of Kimura's disease and the patient was shifted to appropriate treatment with oral prednisone.

Conclusion: Kimura's disease is rare and may be confused with other diseases such as malignancy or tuberculosis. Histopathologic diagnosis is necessary as its treatment differs from tuberculosis and other diseases.

\section{Keywords: Kimura's disease, parotid gland tumor, angiolymphoid hyperplasia with eosinophilia}

Neck masses are among the most common clinical findings to confront any physician, more so an otolaryngologist. Although most masses are benign, malignant processes must still be considered. Infectious causes of neck masses such as tuberculosis are constantly included in the differential diagnosis especially in the Asia-Pacific where it is still prevalent. However, in rare instances, knowledge of uncommon diseases should also be kept in mind. This case illustrates the rare occasion when a common disease entity is considered but turns out to be an unusual diagnosis.

\section{CASE REPORT}

A 30-year-old Micronesian male presented with a gradually-enlarging, painless right infraauricular mass that he noted to measure about the size of a marble six years prior to consult, without any other associated symptoms. A fine-needle aspiration biopsy of the mass revealed Pleomorphic Adenocarcinoma and he was advised surgical excision but was lost to follow-up.

One year prior to consult, the patient experienced progressive enlargement of the right infraauricular mass with numbness over the area and weight loss but did not consult until one month prior to admission when the size of the mass had increased to approximately $8 \times 8 \times 5 \mathrm{~cm}$ 
with pain and numbness in the area, limitation of neck motion and easy fatigability. He denied any dyspnea, dysphagia, sore throat, hoarseness or fever. Review of systems was unremarkable except for weight loss and anorexia. His past medical, family and surgical history were noncontributory. He was previously a construction worker with a smoking history of 2 pack-years, occasional intake of alcoholic beverages and no history of illicit drug use.

Physical examination revealed a soft, fluctuant, non-tender, nonhyperemic right infra-auricular mass measuring approximately $11 \times 8 \mathrm{x}$ $5 \mathrm{~cm}$ with no induration or fixation to underlying structures. (Figure $1 \mathrm{~A}$ and $1 B$ ) Multiple $1 \times 1 \mathrm{~cm}$ non-tender cervical lymphadenopathies were also noted in the right side of the neck at level II. He had a hyperemic posterior pharyngeal wall without tonsillar enlargement but anterior rhinoscopy and otoscopy were unremarkable and there were no lesions on the external ear and face. The initial assessment was parotid mass, rule out malignancy.

Complete blood count, urinalysis, fasting blood sugar, lipid profile and creatinine were unremarkable except for neutrophilia at $20.6 \mathrm{x}$ $10 \wedge 9 / \mathrm{L}$ (reference range $4.5-10$ ) and eosinophilia at 0.5 (reference range $0.01-0.04$ ). Chest x-ray and Computed Tomography (CT) scans of the chest and abdomen were unremarkable. Contrast-CT of the neck showed a diffusely-enlarged heterogenous right parotid gland with multiple varied sized nodules while the left parotid gland was normal in size with multiple nodules. (Figure 2) Multiple cervical lymphadenopathies were noted. (Figure 3) Nodular fullness of the nasopharynx and right side of the oropharynx and tonsillar region was also reported. (Figure 4) The differentials at this point were lymphoma, tuberculosis and metastatic lymphadenopathy either from a primary malignancy in the parotid gland or nasopharynx.

Flexible nasal endoscopy confirmed the CT scan findings of nasopharyngeal mass seen more on the right side. A nasopharyngeal biopsy yielded chronic inflammation and a tissue Acid-fast Bacilli (AFB) smear of the nasopharyngeal mass was negative. However, Tuberculosis (TB) Polymerase Chain Reaction (PCR) of the same specimen was positive, hence, tuberculosis chemotherapy was started.

Fine needle aspiration biopsy of the right parotid was inconclusive, and incisional biopsy under general anesthesia revealed a $10 \mathrm{~cm}$ mass with caseous material and a soft, well-encapsulated $3 \mathrm{~cm}$ deep cervical lymph node. Pathologic gross description of the lymph node was a reddish-pink, ovoid, flat, doughy tissue measuring $2.5 \times 2.4 \times$ $1 \mathrm{~cm}$ with a yellowish-pink mucoid glistening cut surface. The right parotid mass was grossly described as an irregularly ovoid yellowbrown granular doughy tissue measuring $3.0 \times 2.0 \times 1.3 \mathrm{~cm}$ with a pink to brown smooth homogenous cut surface. Purified Protein Derivative
(PPD) skin tests showed negative results at $3 \mathrm{~mm}$ with no induration. Histopathologic examination of both specimens demonstrated reactive germinal follicles with dense eosinophilic cell infiltrates (Figure 5, 6, 7) diagnosed as angiolymphoid hyperplasia with eosinophilia, otherwise known as Kimura's disease. A follow-up blood examination of total lgE revealed $>5,000 \mathrm{kU} / \mathrm{L}$ (reference range $25-100 \mathrm{kU} / \mathrm{L}$ ). Surgical excision was recommended but the patient opted for more conservative management and he was started on oral prednisone at $40 \mathrm{mg} /$ day in two divided doses over a 4-week tapering dose. There was an interval decrease in the size of the mass after three days of treatment and the patient was subsequently discharged but lost to follow-up.

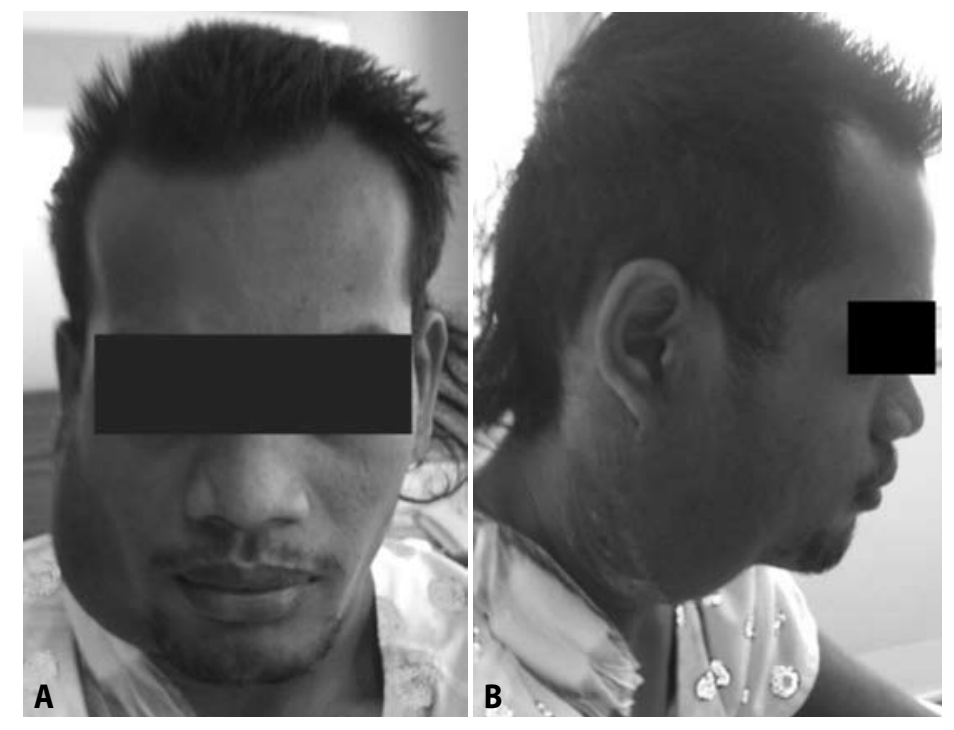

Figure 1. A. Anterior view of the patient showing the mass at the right infraauricular area. B. Lateral view

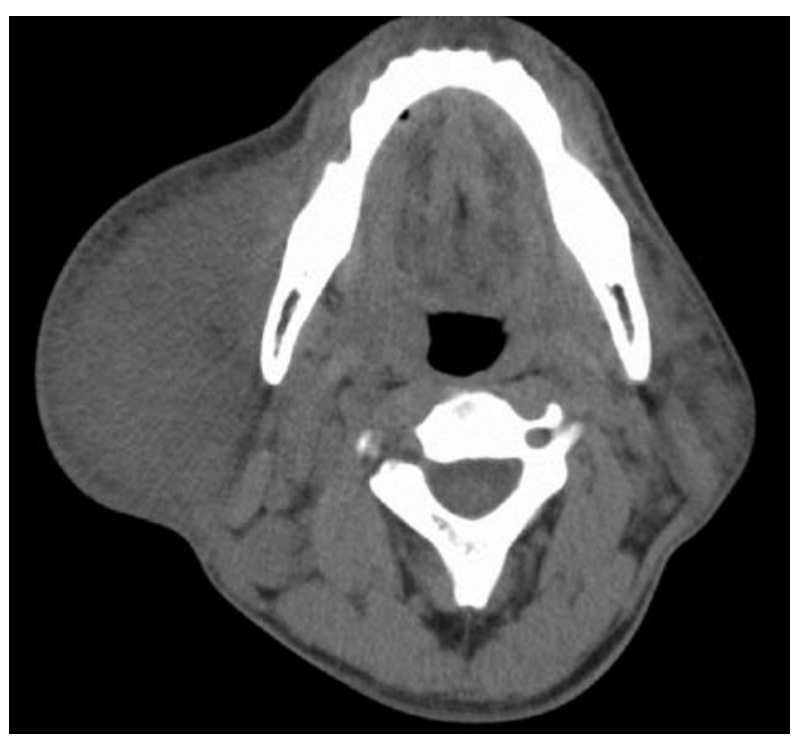

Figure 2. Contrast axial CT scan of the neck showing a diffusely-enlarged heterogenous right parotid gland with multiple varied sized nodules and a normal-sized left parotid gland. 


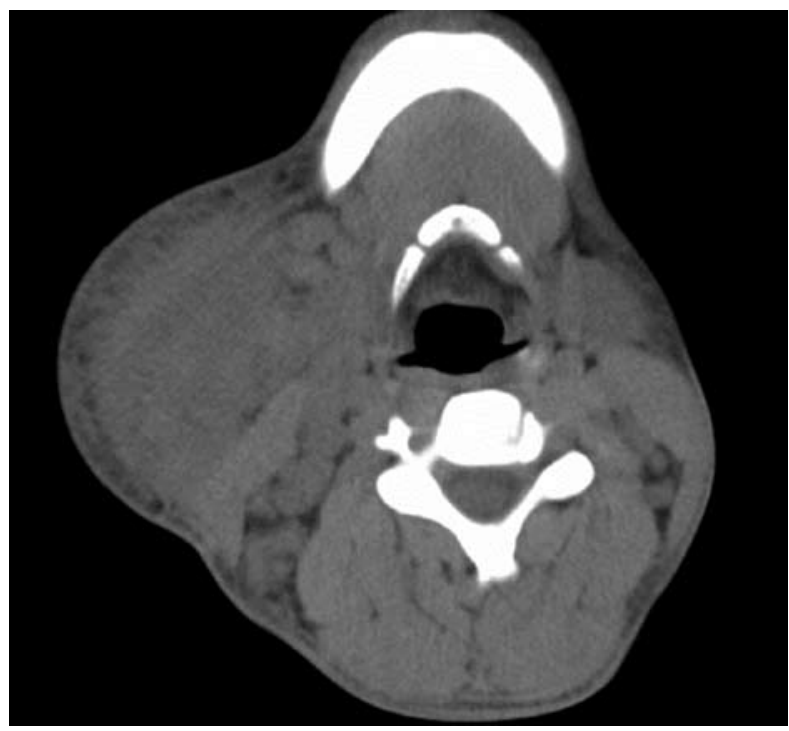

Figure 3. Multiple cervical lymphadenopathies seen in axial contrast CT scan of the neck at the level of the hyoid.

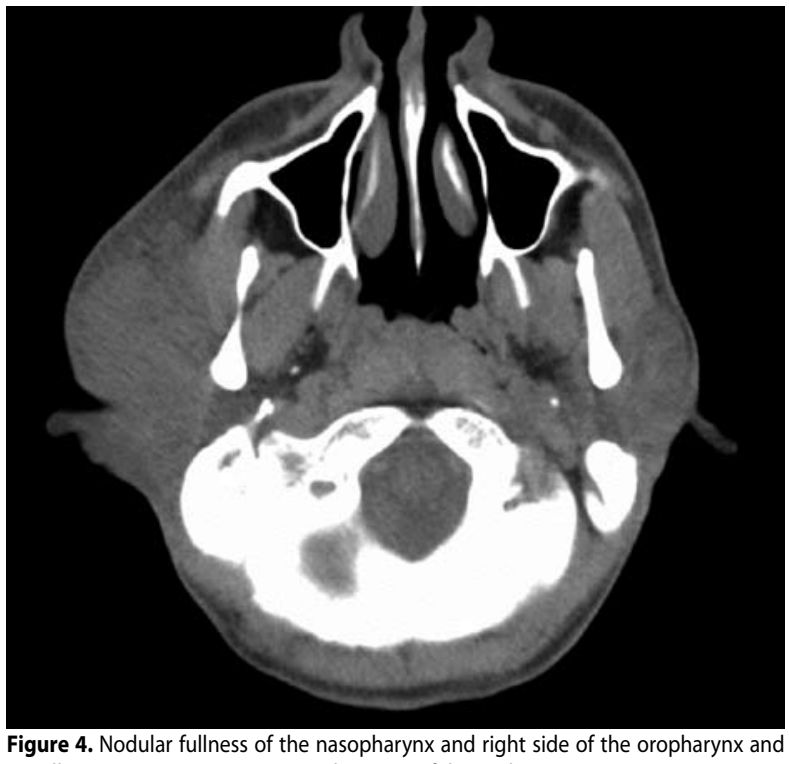
tonsillar region seen in contrast axial CT scan of the neck.

\section{DISCUSSION}

The differential diagnosis for neck masses is extremely broad, making it necessary to categorize patients according to age group and location of the mass to narrow down other considerations. Categorizing patients according to age group and location of the mass is helpful in narrowing the differential diagnosis.' The differential diagnosis of patients presenting with neck masses in the lateral neck area, in the age group of 16-40 years old include inflammatory conditions such as viral or bacterial adenitis, sialadenitis and granulomas; neoplasms such as lymphomas, sarcomas, parotid carcinomas and metastasis and other infectious processes such as tuberculosis. ${ }^{1,2}$
In this case, the patient was a 30-year-old Micronesian male presenting with the triad of painless slowly growing enlarging soft tissue mass, associated lymphadenopathy and peripheral eosinophilia. The presentation in this case could be easily mistaken for a primary parotid gland tumor, lymphoma, metastasis or tuberculosis. Although a high index of suspicion is required and malignancy should be considered if presented with a head and neck mass, ${ }^{3}$ parotid malignancy and lymphoma were ruled out based on the absence of symptoms such as facial nerve paralysis, rapid growth of mass, fever and loss of appetite.

In a study by Henry and Burnett in 1978, the incidence of ALHE within racial groups was categorized into 115 Orientals, 46 whites, 7 blacks and 3 Middle Eastern patients. To our knowledge, there have been only four published reports of ALHE occurring in Filipinos as of 2009. One case report occurred in a 26-year-old Filipino male presenting with multiple reddish-brown papules and nodules over the left external ear. ${ }^{4}$ Another report involved a 15-year-old Filipino male with a 9-year history of a corn-sized mass on his left earlobe. ${ }^{5}$ The third report entailed a 32-yearold man with a slowly growing right upper neck mass accompanied by multiple subcutaneous nodules for two years. ${ }^{6}$ The fourth report was mentioned by Soeria-Atmadja et al. from Karolinska University Hospital in Sweden ${ }^{7}$ but was not included in this report.

Routine blood tests help rule out inflammatory causes while imaging studies aid in the location and size of tumors. Laboratory findings for this patient included peripheral eosinophilia, which gives a clue to the diagnosis of Kimura's disease. ${ }^{2}$ This finding can be further confirmed by an elevated serum IgE level. Imaging studies can also support the diagnosis of KD but may not always do so as the case of a 21-year-old Caucasian male with an initial clinical assessment of Lymphangioma or Hemangioma. Intra-operatively, the mass was so described:

A characteristic appearance was the adherence of the skin to the parotid mass, with no clear plane of dissection between the lesion and the parotid tissue. The mass was not cystic but solid opposing the preoperative palpation findings. ${ }^{3}$

The diagnostic challenge of KD can be solved with histologic study, preferably from a lymph node biopsy. Fine needle aspiration cytology is helpful but often inconclusive. Histologic examination of the excised tissue remains the more diagnostic approach. ${ }^{3,8}$ In this patient, definitive diagnosis was only confirmed after histopathologic study of the right infraauricular mass. Malignancies such as pleomorphic adenocarcinoma or Hodgkin's lymphoma were ruled out as histopathologic findings did not reveal any atypical cells, lymphocyte predominance or malignant change.

Kimura's disease is a rare entity that usually occurs sporadically in 


\section{CASE REPORTS}

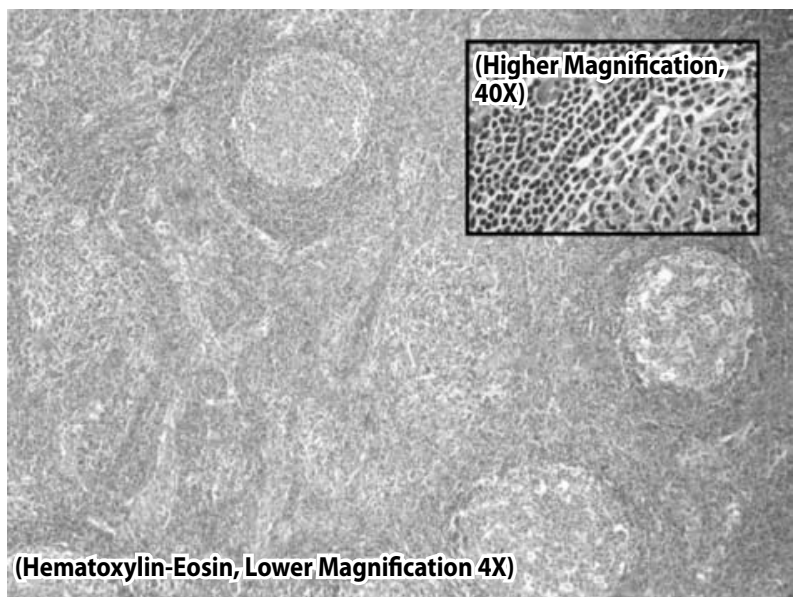

Figure 5. Cervical lymph node, (H\&E Stain) Lower magnification (4X) shows reactive germinal follicles with dense eosinophilic cell infiltrates. (Inset) Higher magnification (40X).

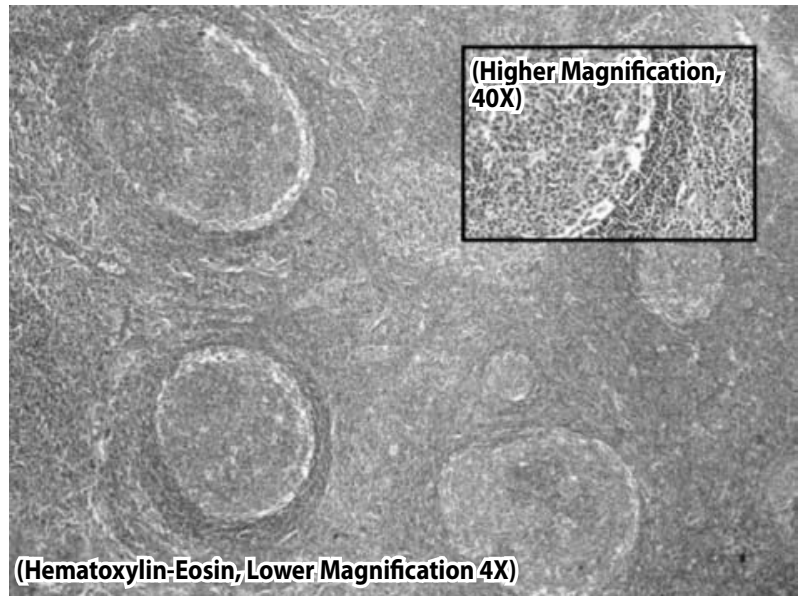

Figure 6. Cervical lymph node, (H\&EStain) Lower magnification (4X) shows reactive germinal follicles with dense eosinophilic cell infiltrates. (Inset) Higher magnification $(40 \mathrm{X})$.

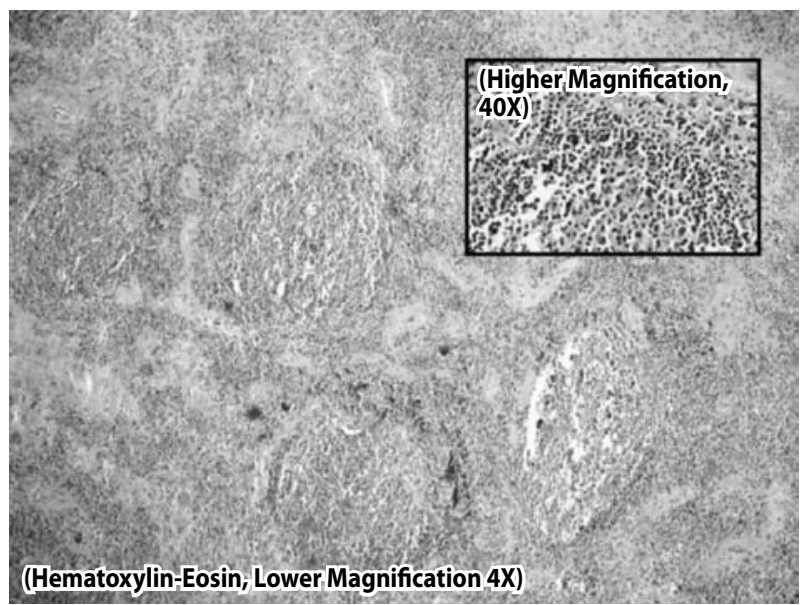

Figure 7. Parotid mass, right. (H\&E Stain). Lower magnification $(4 \mathrm{X})$ shows reactive germinal follicles with dense eosinophilic cell infiltrates. (Inset) Higher magnification (40X).
Western countries but is endemic in Asia. It was first described in 1937 in the Chinese literature but the definitive histological description was published by Kimura, et al. in $1948 .{ }^{8}$ Clinical presentation is varied and may fluctuate for several years. Kimura's Disease usually presents with solitary or several subcutaneous nodules located in the head and neck which sometimes can be painful and pruritic that slowly increase in volume accompanied by satellite adenopathies and/or increase volume of the salivary glands. ${ }^{9}$ Although the masses enlarge slowly, the patients remain otherwise asymptomatic. It can also present with symptoms of cough, massive hemoptysis and lymph nodes similar to tuberculosis. ${ }^{10}$

Histopathologic examination findings are the same regardless of the site of involvement, and are characterized by lymphoid follicle formation with prominent germinal centres, infiltration of eosinophils, sometimes forming microabscesses, fibrosis, increased post-capillary venules and vascular proliferation. ${ }^{8,9}$ Constant features of KD are preserved lymph node architecture, florid germinal centers, eosinophilic infiltration and increased amount of post-capillary venules. The single rare feature is progressive transformation of the germinal center. ${ }^{6,11}$

Several authors have used the terms ALHE and KD interchangeably due to similarities in their clinical and pathologic presentations. Even in this study, the final histopathologic result released stated that the diagnosis is angiolymphoid hyperplasia with eosinophilia, otherwise known as Kimura's disease. However, in a review of seven cases, Salal AlJittawa et al. attempted to distinguish these two entities. As described, Kimura's Disease usually presents with subcutaneous nodules located in the head and neck more commonly seen in men in the second to third decade. ${ }^{912}$ Prominent manifestations are blood eosinophilia and regional lymphadenopathy with long-standing history averaging 9.6 years. Histologic features primarily show lymphoid follicle formation with prominent germinal centres, infiltration of eosinophils, lymphocytes, plasma cells and mast cells with variable degree of vascular proliferation.

On the other hand, ALHE can be either dermal or subcutaneous located in the head and neck mostly seen in women from the second to the fifth decade. Blood eosinophilia and regional lymphadenopathy appear less frequently. Histologically, vascular proliferation is more prominent with infiltrates similar to KD but with less fibrosis and lymphoid follicle formation owing to its shorter duration of 2 to 3 years. $8,9,12$ Based on this differentiation, the demographic features of this case in a 30-year-old male with a 6-year history of lymphadenopathy in the head and neck and blood eosinophilia point more towards the diagnosis of KD.

Optimal treatment of KD is not well established but is aimed to preserve cosmesis and prevent recurrences and long-term sequelae. The range of treatment options includes conservative management, 


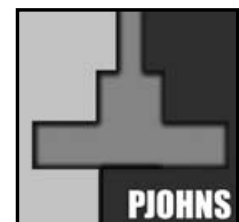

CASE REPORTS

Philippine Journal Of Otolaryngology-Head And Neck Surgery

VOL. 27 No. 1 JANUARY - JUNE 2012

oral corticosteroids, radiotherapy, cryotherapy, laser fulguration and surgical excision. Other therapeutic options include cytotoxic agents, cyclosporine and pentoxifyline but with variable results. ${ }^{13,14}$

The diagnosis of Kimura's disease is highly difficult and misleading. It is indeed a diagnostic and therapeutic challenge. This report emphasizes the importance of having a high index of suspicion in an Asian patient presenting with a head and neck mass and reiterates the need of becoming acquainted with this disease to prevent subsequent complications due to delays in diagnosis and treatment.

ACKNOWLEDGEMENTS

We would like to thank Agustina Abelardo, M.D., our pathologist and scientific advisor; and Mr. Mark James G. Cayabyab who helped in the review of literature.

\section{REFERENCES}

1. Cummings CW, Haughey BH, Thomas JR, Harker LA, Flint PW. Cummings otolaryngology head \& neck surgery. $4^{\text {th }}$ ed. Pennsylvania: Mosby; 2005.

2. Roufosse FE, Goldman M, Cogan E. Hypereosinophilic syndromes. 2007 September 11 [Accessed 2010 March 1] Available from http://www.ncbi.nlm.nih.gov/pmc/articles/PMC2045078/ pdf/1750-1172-2-37.pdf

3. Can iH, Guduz V, Pulat H, Samim E. Kimura's disease in the parotid gland. Internet J Head Neck Surg. 2009 February 13 [Accessed 2010 March 1] Available from http://www.ispub.com/ostia/ index.php?xmlFilePath=journals/ijhns/vol1n2/kimura.xml

4. Roxas-Rosete C, Tianco E, King-Ismael D. Angiolymphoid hyperplasia with eosinophilia in a Filipino man. Int J Dermatol. 1995 Apr; 34(4): 267-70.

5. Rossi H, Dy W. Cancer and keloid or Kimura and steroid. Philipp J Otolaryngol Head Neck Surg 1990; 5: 34-38.

6. Jani A, Coulson M. Kimura's disease: a typical case of a rare disorder. West J Med 1997 Feb; 166(2):142-144

7. Soeria-Atmadja S, Oskarsson T, Celci G, Sander B, Berg U, Gustafsson B. Maintenance of remission with cyclosporine in paediatric patients with Kimura冈s disease - two case reports. Acta Paediatrica, 2011 100: e186-e189. doi: 10.1111/j.1651-2227.2011.02259.x

8. Viswanatha B. Kimura disease: An unusual cause of head and neck masses. Report of 2 cases. Ear, Nose Throat J. 2010 Feb; 89(2): 87-89.

9. KuoTT, Shih LY, Chan HL. Kimura's disease. Involvement of regional lymph nodes and distinction from angiolymphoid hyperplasia with eosinophilia. Am J Surg Pathol 1988 Nov; 12(11):843-54.

10.Rajaver S, Acharya V, Rau A, Sivaramakrishnan C, Sahoo RC, Lobo F. A rare case of Kimura's disease presenting with cough, hemoptysis and axillary lymph node involvement. Internet $J$ Pulm Med 2009; 11:1.

11.Googe PB, Harris NL, Mihm MC Jr. Kimura's disease and angiolymphoid hyperplasia with eosinophilia: two distinct histopathological entities. J Cutan Pathol 1987 Oct; 14(5): 263-71

12. Al-Jittawi S, Oumeish O. Angiolymphoid hyperplasia with eosinophilia. Int/ Dermatol 1989: 28: 114-118.

13. Hui PK, Chan JK, Ng CS, Kung IT, Gwi E. Lymphadenopathy of Kimura's disease. Am J Surg Pathol 1989 Mar; 13(3):177-186.

14. Yuen HW, Goh YH, Low WK, Lim-Tan SK. Kimura's disease: diagnostic and therapeutic challenge. Singapore Med J 2005 Apr; 46(4): 179-183. 